\title{
Biomarkers of adiposity are elevated in preterm very-low-birth-weight infants at 1, 2, and 3 y of age
}

\author{
Andrea F. Duncan', Joshua A. Frankfurt ${ }^{2}$, Roy J. Heyne ${ }^{2}$ and Charles R. Rosenfeld ${ }^{2}$
}

BACKGROUND: Preterm, very-low-birth-weight (PT-VLBW) neonates are at-risk for metabolic syndrome later in life. At 1-3 $y$, they exhibit excessive weight-for-length $z$-scores $\left(W_{t}-L_{z}\right)$ and elevated systolic blood pressures (SBP). Serum adipokines are biomarkers of adiposity, but expression in PT-VLBW infants is unclear. We examined the correlation between serum adipokine levels, anthropometric measures and SBP in PT-VLBW neonates at follow-up.

METHODS: This was a cross-sectional cohort study of PT-VLBW infants at 1, 2, and 3 y of age (40/cohort). We measured SBP, abdominal circumference (AC) and anthropometrics; calculated age/gender-specific z-scores for Wt, L, Wt-L and subscapular skin fold (SS $)$, and measured serum adipokines.

RESULTS: Serum leptin was unaffected by chronologic age and gender, but was positively correlated with weight, $\mathrm{Wt}-\mathrm{L}_{\mathrm{z}^{\prime}}$ $\mathrm{AC}$, and $\mathrm{SS}_{7}$ at 1 and 3 y $(P<0.01)$. Female infants at 1 and 3 y had a more significant relationship than males between serum leptin and $\mathrm{SS}_{7}(P<0.001, R=0.75$ and $P<0.001, R=$ 0.70 , respectively). Adiponectin levels were $16-20 \%$ lower at 3 vs. $1-2$ y $(P=0.02$, ANOVA $)$ and negatively correlated with SBP. CONCLUSION: Although serum leptin was unrelated to advancing age, gender, and SBP in PT-VLBW infants, levels correlated with measures of adiposity at 1 and $3 y$, females > males, suggesting leptin resistance may occur in early infancy.

nfants born very preterm (PT; $\leq 32 \mathrm{wk}$ of gestation) and verylow-birth-weight (VLBW; birth weight $(\mathrm{BW}) \leq 1,500 \mathrm{~g}$ ) are at increased risk of obesity, metabolic syndrome (MBS), and hypertension later in life compared to their term counterparts (1-3). They have reduced insulin sensitivity, which persists after correcting for appropriateness of intrauterine growth (2). In addition, women born PT ( $\leq 37 \mathrm{wk}$ of gestation) are at greater risk of metabolic derangement than men (3). Although altered fetal programming may contribute to the pathogenesis of these findings (4), postnatal variables such as disordered nutrition and growth may also contribute to reprogramming, leading to subsequent metabolic derangements including MBS, obesity, and cardiovascular disease (4-6). For example, term infants with rapid disproportionate weight-for-length (Wt-L) growth between birth and 6 mo have elevated systolic blood pressures (SBP) at $6 \mathrm{mo}$ and $3 \mathrm{y}$ of age (7). Children born PT-VLBW also have evidence of growth derangements after birth and in early childhood (7-9). At 1-3 y of age, they exhibit excessive Wt-L $z$-scores $\left(\mathrm{Wt}-\mathrm{L}_{\mathrm{z}}\right)$, i.e., relative obesity, and elevated SBP and $\operatorname{SBP} z$-scores $\left(\mathrm{SBP}_{\mathrm{Z}}\right)(8,9)$. However, their risk for subsequent MBS and hypertension is unclear.

Though reprogramming may contribute to the long-term metabolic abnormalities, hypertension and cardiovascular risk in PT-VLBW, the pathogenesis is unclear. Alterations in adiposity and thus adipokine synthesis may contribute, but are poorly studied. Adipokines are secreted by adipocytes (10), and increases in fat mass results in dysregulated secretion. This promotes inflammation and development of cardiovascular and metabolic disorders (11). Though there are several adipokines, their association with MBS and cardiovascular risk are best defined for leptin, adiponectin, and resistin (11). In adults, serum leptin levels increase with increasing adiposity, providing a novel biomarker for adiposity. Leptin is proinflammatory and increased serum levels correlate with insulin resistance, arterial stiffness, elevated blood pressure, and increased cardiovascular risk (10-12). Leptin, therefore, may play a central role in the association between obesity, MBS, and cardiovascular disease through development of leptin resistance at the hypothalamic-pituitary axis in adults (13-16). In contrast, serum adiponectin levels are inversely related to adiposity, considered anti-inflammatory, and elevations are associated with lower blood pressure and decreased arterial stiffness $(11,12,16,17)$. Adults with hypertension and obesity exhibit decreased levels of adiponectin. Circulating resistin levels also may be increased in obesity. Thus, adipokines are novel biomarkers of MBS and subsequent cardiovascular disease $(11,16,18,19)$.

Though adipokines are important biomarkers of obesity, MBS, and cardiovascular risk in adults $(13,14,20)$, little is known about their homeostasis in PT-VLBW infants in the first years after birth. It is unclear if the pattern of postnatal growth, changes in adiposity, and presence of elevated SBP correlate with altered serum adipokine levels. Thus, we examined the relationships between postnatal growth, SBP and circulating adipokine levels in PT-VLBW infants at 1, 2, and $3 \mathrm{y}$

'Department of Pediatrics, Division of Neonatal-Perinatal Medicine, University of Texas Health Science Center, Houston, Texas; ${ }^{2}$ Department of Pediatrics, Division of Neonatal-Perinatal Medicine, University of Texas Southwestern Medical Center, Dallas, Texas. Correspondence: Charles R. Rosenfeld (charles.rosenfeld@utsouthwestern.edu) Received 5 July 2016; accepted 30 December 2016; advance online publication 5 April 2017. doi:10.1038/pr.2017.11 
Table 1. Demographic data for study subjects in each cohort

\begin{tabular}{|c|c|c|c|c|}
\hline & All cohorts $(n=120)$ & Cohort $1(n=40)$ & Cohort $2(n=40)$ & Cohort $3(n=40)$ \\
\hline Mean age at visit (years) & & $0.81^{\mathrm{a}}(0.72-0.97)^{*}$ & $1.8^{b}(1.7-2.2)$ & $2.8^{\complement}(2.7-3.6)$ \\
\hline \multicolumn{5}{|l|}{ Race (\%) } \\
\hline Black & 24 & 35 & 20 & 17 \\
\hline White & 1 & 2.5 & 0 & 0 \\
\hline \multicolumn{5}{|l|}{ Sex $(\%)$} \\
\hline Male & 48 & 47 & 42 & 55 \\
\hline Female & 52 & 53 & 58 & 45 \\
\hline \multicolumn{5}{|l|}{ Birth information } \\
\hline Maternal hypertension (\%) & 32 & 30 & 33 & 33 \\
\hline Small for gestational age (\%) & $23(19)^{\dagger}$ & $9(23)$ & $8(20)$ & $6(15)$ \\
\hline Ponderal index & $2.2 \pm 0.3$ & $2.2 \pm 0.3(1.7-2.8)$ & $2.2 \pm 0.3(1.7-3.1)$ & $2.1 \pm 0.3(1.6-2.9)$ \\
\hline$<10^{\text {th }}$ centile $(\%)$ & $14(12)$ & $5(13)$ & $3(8)$ & $6(15)$ \\
\hline \multicolumn{5}{|l|}{ Socioeconomic status } \\
\hline Median family income & $\$ 16-24,000$ & $\$ 16-24,000^{\mathrm{a}}$ & $\$ 12-15,999^{b}$ & $\$ 12-15,999^{b}$ \\
\hline Number of people in home & 4.7 & 5.0 & 4.7 & 4.5 \\
\hline Mean caregiver education (years) & $9.9 \pm 3.5$ & $10.7 \pm 3.9(2-16)$ & $9.0 \pm 3.5(1-14)$ & $10.0 \pm 3.0(6-16)$ \\
\hline
\end{tabular}

Different superscripts across a row represent significant differences between cohorts when analyzed by one-way ANOVA followed by multiple comparison procedure, $P<0.05$.

*Range of values within cohorts are in parenthesis.

**Data are mean \pm SD.

$\vee$ alue in parenthesis is the per cent of infants within a cohort.

of chronological age. We hypothesized that measures of adiposity and elevations in SBP would correlate with increases in serum leptin and resistin levels and decreases in serum adiponectin. The studies were considered descriptive and thus, hypothesis-generating.

\section{METHODS}

\section{Population}

PT-VLBW infants $(n=120)$ who were born at Parkland Hospital and subsequently received their primary outpatient care in the Low Birth Weight Clinic at Children's Medical Center of Dallas between 2007 and 2008 were consecutively enrolled after obtaining informed consent. They were entered into a cross-sectional cohort study at 1,2 , or 3 y chronological age ( $n=40 /$ cohort). These infants were included in recent studies of blood pressure and renal function $(9,21)$. Exclusion criteria included major congenital anomalies, congenital adrenal hyperplasia, and short bowel syndrome requiring total parenteral nutrition after hospital discharge. The Institutional Review Boards of the University of Texas Southwestern Medical Center at Dallas and Children's Medical Center of Dallas approved the study.

\section{Measurements}

After obtaining consent, we measured Wt, L, subscapular skin-fold thickness (SS) and abdominal circumference (AC) and converted Wt, $\mathrm{L}, \mathrm{Wt}-\mathrm{L}$ proportion and SS to age and gender specific $z$-scores $\left(\mathrm{Wt}_{\mathrm{Z}}\right.$, $\mathrm{L}_{\mathrm{Z}}$, Wt- $\mathrm{L}_{\mathrm{Z}}$, and $\mathrm{SS}_{\mathrm{Z}}$, respectively) using reference data from the World Health Organization Anthro calculator (22-24). Adjusted age at the time of measurement was used for this calculation and determined by subtracting weeks born preterm, i.e., (40 completed weeks of gestation at birth)-(chronologic age on the visit date). SBP was measured as previously described $(8,9)$.

\section{Assays}

Blood samples $(\sim 1.5 \mathrm{ml})$ collected from each infant at the time of the clinic visit were immediately centrifuged at $10,000 \times \mathrm{g}$ and the serum stored at $-80{ }^{\circ} \mathrm{C}$ until the time of assay. Samples were available for measurement of adipokines in $96 \%$ of infants, 36/40 in Group 1, 40/40 in Group 2, and 39/40 in Group 3. At the time of assay, samples were thawed, diluted 1:4 and 1:200 to capture all analytes on their respective standard curves and measured in duplicate. One sample diluted at 1:4 was included in each assay to calculate intra- and interassay variability. Assays included IL-6, TNF $\alpha$, leptin, adiponectin, and resistin measured by ELISA with Fluorokine MultiAnalyte Profiling kits from R\&D Systems (Minneapolis, MN) and using the BioPlex 2200 Analyzer (Bio-Rad Laboratories, Hercules, CA). Intra-assay and interassay coefficients of variability were $11.4 \%$ for leptin, $10.5 \%$ for adiponectin, and $12.9 \%$ for resistin. IL- 6 and TNF $\alpha$ were below sensitivity of the assay in all samples.

\section{Statistical Analyses}

Data for age groups were compared using one-way ANOVA for continuous variables; $\chi^{2}$ and Fisher's exact tests were used to compare categorical variables. The effect of gender within cohorts was determined using two-way ANOVA. When the ANOVA was significant $(P$ $<0.05$ ), Dunn's multiple comparison procedures were used to compare groups. Correlations between serum adipokines and SBP and various growth parameters were determined using linear regression analyses. Data are means \pm SD unless otherwise noted. 


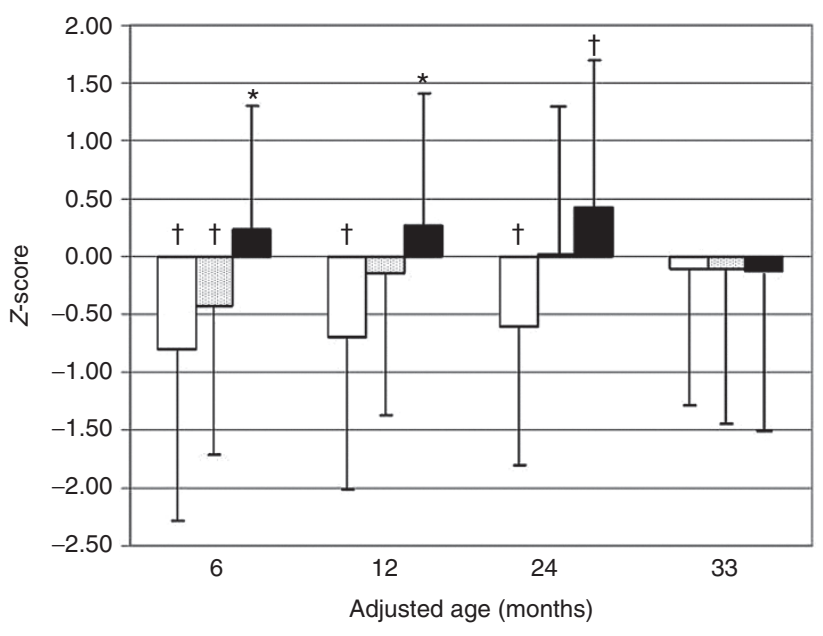

Figure 1. Changes in length (open columns), weight (stippled columns), and weight-for-length (filled columns) $z$-scores in PT-VLBW infants at 6 ( $n=$ $120), 12(n=120), 24(n=80)$, and $33 \mathrm{mo}(n=40)$ chronologic age. ${ }^{\dagger} P<$ 0.01 and ${ }^{*} P<0.05$ compared to the normal distribution.

\section{RESULTS}

Infants were enrolled between May and September 2007. The majority were Hispanic, reflecting the demographics of the delivery population at Parkland Hospital during the study period (Table 1). There were no differences in gender distribution between cohorts. The fall in mean BW across cohorts paralleled a fall in mean gestational age at birth. Nineteen percent of infants had a BW $\leq 10^{\text {th }}$ centile corrected for gestational age and were small for gestational age (SGA). Twelve percent had asymmetrical fetal growth restriction with a Ponderal Index $\leq$ $10^{\text {th }}$ centile; the distribution was similar across cohorts.

\section{Growth}

Because of the cohort design and the timing of preset clinic visits, we collected additional growth measurements at 6 mo $(n=120), 12 \mathrm{mo}(n=120)$, and $24 \mathrm{mo}(n=80)$ adjusted age, and included them in the growth analyses to better characterize the anthropometric changes occurring in this population compared to published growth data for healthy term infants (22-24). There were differences in anthropometric measurements at each age compared to expected (Figure 1). At $6 \mathrm{mo}^{-\mathrm{Wt}_{\mathrm{z}}}$ and $\mathrm{L}_{\mathrm{Z}}$ were significantly less than expected, whereas $\mathrm{Wt}-\mathrm{L}_{\mathrm{Z}}$ was greater than expected, suggesting excessive Wt gain relative to length in all study infants. This was also true for all cohorts at 12 mo; however, the difference in $\mathrm{Wt}_{\mathrm{z}}$ was less while $\mathrm{L}_{\mathrm{Z}}$ remained quite low, resulting in $\mathrm{Wt}-\mathrm{L}_{\mathrm{z}}$ greater than expected. At $24 \mathrm{mo}$, the combined 24 and 33 mo cohorts exhibited a lesser difference in $\mathrm{Wt}_{\mathrm{z}}$ compared to the reference population, but minimal change in $\mathrm{L}_{\mathrm{Z}}$; thus, Wt- $\mathrm{L}_{\mathrm{Z}}$ continued to be significantly greater. These differences were absent in the 3 y cohort.

\section{Serum Adipokines}

Serum levels of IL-6 and TNF $\alpha$ were below sensitivity of the assay in all samples. CRP did not differ between cohorts, $0.06 \pm 0.1,0.05 \pm 0.1$, and $0.06 \pm 0.1 \mathrm{mg} / \mathrm{ml}$ at 1,2 , and $3 \mathrm{y}(P$ $=0.9)$, respectively. Leptin concentrations also did not differ
Table 2. Serum measurements of leptin, adiponectin, and resistin in each cohort

\begin{tabular}{|c|c|c|c|c|}
\hline & $1 \mathrm{y}(n=36)$ & $2 \mathrm{y}(n=40)$ & $3 \mathrm{y}(n=39)$ & $P$ value $^{* *}$ \\
\hline Leptin (ng/ml) & $4.03 \pm 4.1^{*}$ & $3.50 \pm 2.6$ & $2.89 \pm 3.4$ & 0.12 \\
\hline Adiponectin $(\mu \mathrm{g} / \mathrm{ml})$ & $16.3 \pm 6.9^{a}$ & $17.7 \pm 6.6^{a}$ & $13.7 \pm 4.9^{b}$ & 0.02 \\
\hline Resistin (ng/ml) & $7.6 \pm 3.2^{\mathrm{a}}$ & $5.2 \pm 1.9^{b}$ & $5.1 \pm 2.6^{b}$ & $<0.001$ \\
\hline \multicolumn{5}{|c|}{$\begin{array}{l}\text { *Data are mean } \pm S D \text {. } \\
\text { **P-values were determined by one-way ANOVA for multiple groups. Different } \\
\text { superscripts across a row represent significant differences between cohorts when } \\
\text { analyzed by multiple comparison procedures, } P \leq 0.05 \text {. }\end{array}$} \\
\hline
\end{tabular}

between cohorts (Table 2), were unaffected by gender within and across cohorts, and were unrelated to SBP $(P>0.05)$. When serum leptin levels were correlated with anthropometric measurements within each cohort, positive correlations with $\mathrm{Wt}_{\mathrm{z}}$ were observed at 1 and 3 y $(P=0.009, R=0.43, n=36$ and $P<$ $0.001, R=0.65, n=38$, respectively), but not at $2 \mathrm{y}(P=0.2, R=$ $0.20, n=40)$. After correcting for gender, this was only significant for males at $1 \mathrm{y}$, but was significant for both genders at 3 y $(P \leq 0.003)$. Notably, leptin values were positively correlated $(P \leq 0.01)$ with the Wt- $\mathrm{L}_{\mathrm{z}}$ (Figure $2 \mathrm{a}$ ) and $\mathrm{SS}_{\mathrm{z}}$ (Figure $\left.2 \mathbf{b}\right)$ at 1 and $3 \mathrm{y}$, but not at $2 \mathrm{y}(P>0.1, R=0.2$ for both $)$. This was more significant in females vs. males for $\mathrm{Wt}-\mathrm{L}_{\mathrm{z}}$ and $\mathrm{SS}_{\mathrm{z}}$ at 1 and $3 \mathrm{y}$ $(P \leq 0.009$ vs. 0.01 to 0.07$)$. We also compared the leptin levels in infants who had been growth restricted at birth, i.e., SGA or low ponderal index, with normally grown infants by two-way ANOVA. There was no significant difference, $P>0.1$ for either parameter.

Since serum leptin levels generally correlate with $\mathrm{Wt}$, we adjusted values for Wt at the time of measurement and used the leptin/Wt proportion and concentrations $<$ or $\geq 5 \mathrm{ng} / \mathrm{ml}$ (the upper range of normal at this age; 25, 26) to describe the infants with elevated levels. The leptin/Wt values were greater $(P \leq 0.001)$ in infants with levels $\geq 5 \mathrm{ng} / \mathrm{ml}$ in all cohorts (Figure 3). However, as the difference in the $\mathrm{Wt}_{\mathrm{z}}$ fell with increasing chronological age (Figure 1), the number of infants per cohort with leptin values $\geq 5 \mathrm{ng} / \mathrm{ml}$ also was less, decreasing from 10 to 4 .

Adiponectin levels differed across age-related cohorts and were $16-20 \%$ lower in 3 vs. $1-2$ y infants $(P=0.02$, ANOVA; Table 2). There was no gender effect $(P=0.7)$, and values were unrelated to all anthropometric measurements, including BW $(P>0.1)$. There was a weak negative correlation between adiponectin and SBP for the entire study population $(P=0.05, R$ $=-0.19, n=108)$, i.e., SBP fell as adiponectin rose.; However, it was highly significant in the 1 y cohort $(P=0.002, R=-0.49$, $n=36)$ and in both males $(P=0.02, R=-0.56, n=17)$ and females $(P<0.05, R=-0.47, n=19)$ within that cohort.

Resistin levels also were less in 2 vs. 1 y infants and did not differ between 2 and 3 y (Table 2; $P<0.001$ ANOVA). Male values were less than females, $P=0.009$.

\section{DISCUSSION}

Elevated serum leptin levels are associated with adiposity, insulin resistance, and increased cardiovascular risk in adults $(3,10,11)$. PT-VLBW infants are at increased risk of 
a

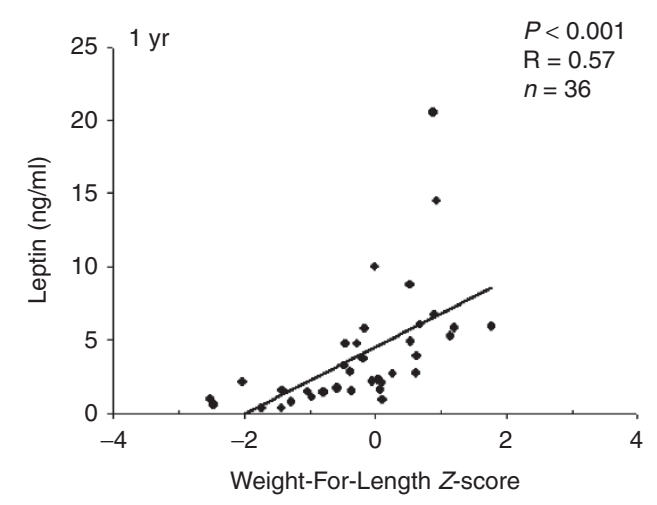

Weight-For-Length Z-score

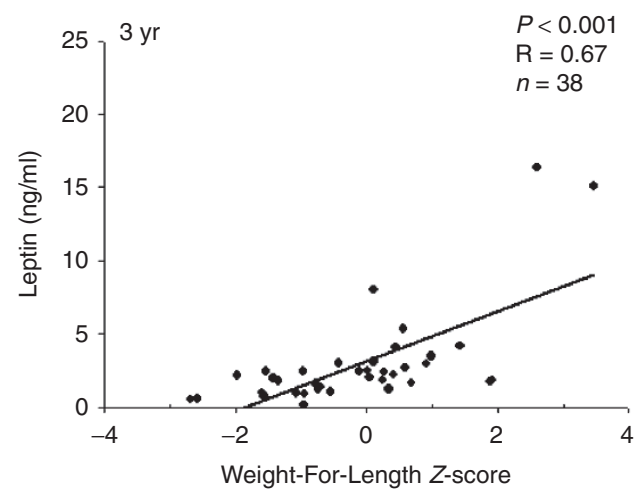

Subscapular Skin-fold Thickness Z-score

b

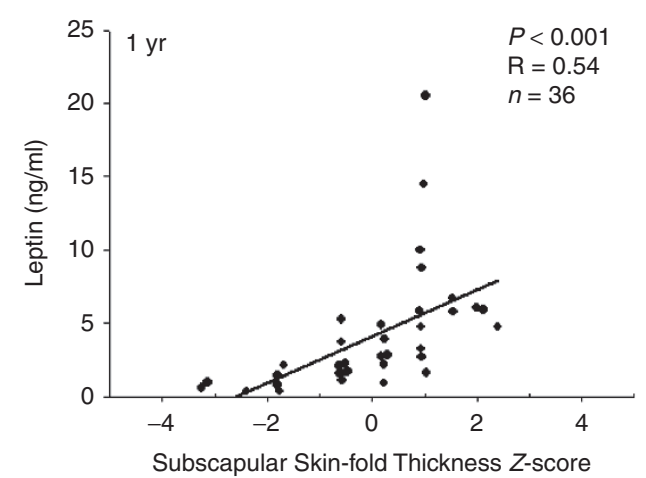

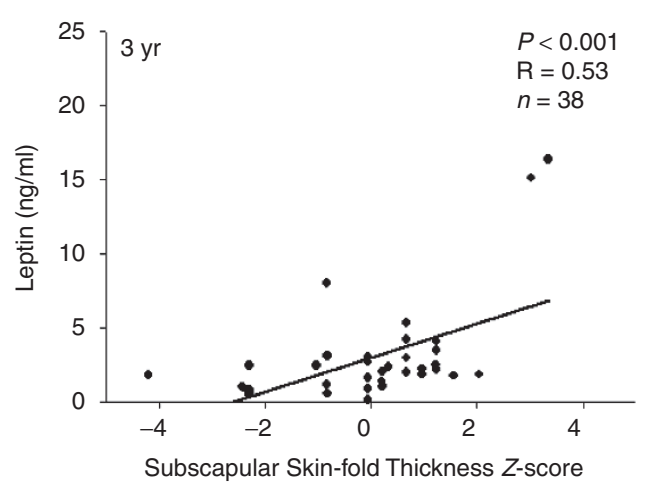

Figure 2. Distribution serum leptin $\geq 5 \mathrm{ng} / \mathrm{ml}$. The correlation between serum leptin concentrations and (a) weight-for-length $z$-scores and (b) subscapular skin-fold thickness z-scores in PT-VLBW infants in cohorts at 1 and 3 y chronologic age. Data were analyzed by linear regression analysis.
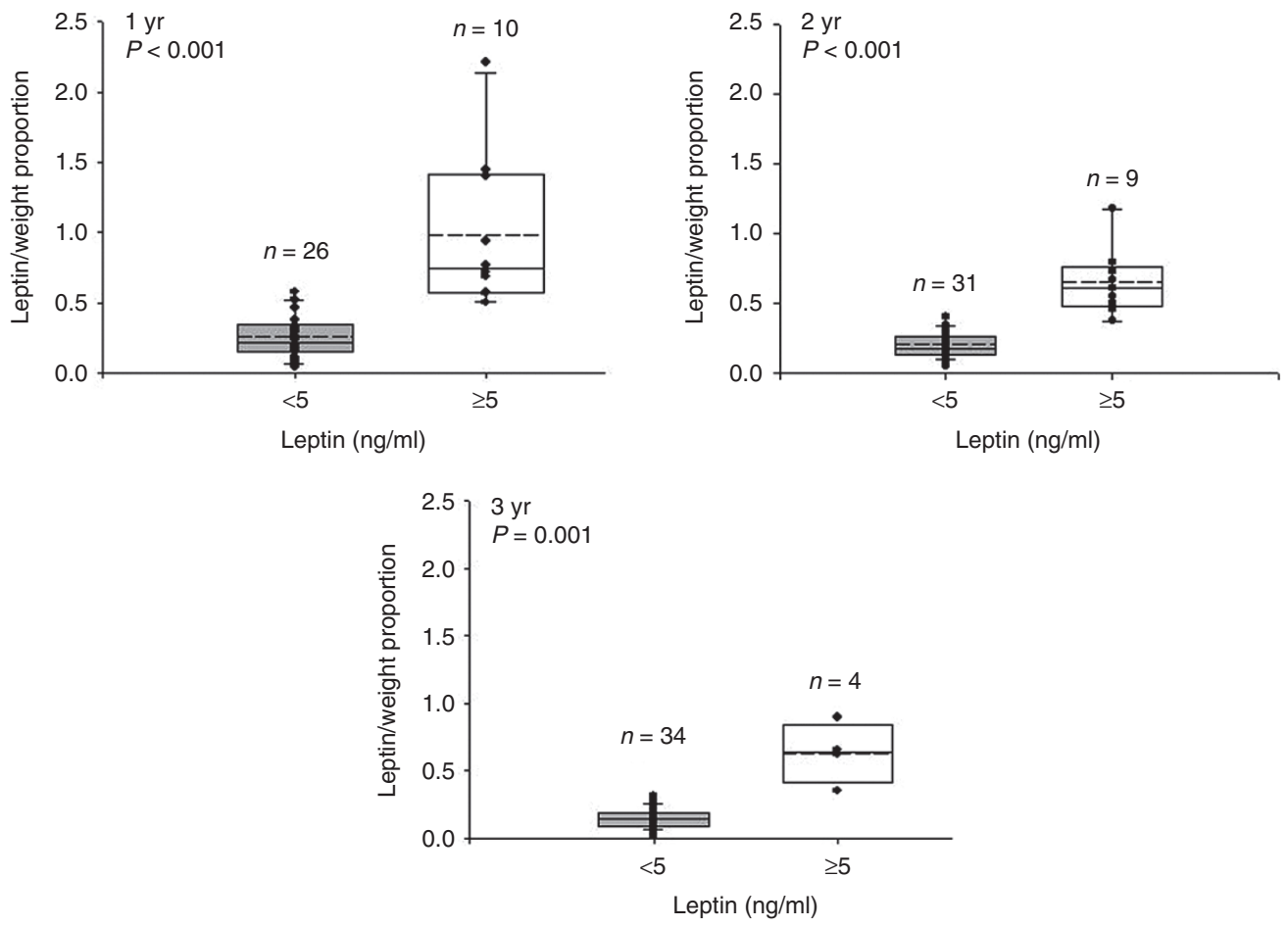

Figure 3. Comparison of serum leptin concentrations corrected for weight using a cutoff of $\geq 5 \mathrm{ng} / \mathrm{ml}$ to distinguish elevated levels in infants within each study cohort at 1, 2, and 3 y. Data were analyzed by $t$-test and represent medians with ranges and SD. 


\section{Articles Duncan et al.}

cardiovascular derangements later in life compared to their term counterparts $(2,3,25)$. This may be due to PT birth and/ or the manner of feeding and growth after birth. We previously reported that PT-VLBW infants grew disproportionately in the first $2 \mathrm{y}$ after birth and demonstrated an elevated $\mathrm{Wt}-\mathrm{L}_{\mathrm{z}}$ and other measures of adiposity (9). Though measures of adiposity in older children and adults are associated with alterations in serum adipokines $(26,27)$, this is unclear in PT-VLBW infants. We now report that, although serum leptin concentrations are unrelated to advancing age and gender, they are positively correlated with measures of adiposity, including increasing $\mathrm{Wt}_{\mathrm{z}}, \mathrm{Wt}-\mathrm{L}_{\mathrm{z}}$, abdominal circumference (AC), and $\mathrm{SS}_{\mathrm{z}}$ in PT-VLBW infant cohorts at $1-3$ y of age. Using a serum leptin value $\geq 5 \mathrm{ng} / \mathrm{ml}$ and the leptin/Wt relationship to characterize these infants, each cohort included a significant number with elevated leptin/Wt values $(28,29)$. There was, however, no relationship between serum leptin levels and SBP. In contrast, serum adiponectin levels decreased across cohorts and were negatively related to SBP. Thus, serum leptin may be a biomarker for adiposity in infancy in PT-VLBW infants and could predict subsequent risk for abnormalities of metabolism, obesity, and cardiovascular risk. This, however, requires further study.

Rapid postnatal growth in term born infants is associated with increases in Wt- $\mathrm{L}_{\mathrm{z}}$ at 6 mo of age, demonstrating disproportionate growth and evidence of early onset adiposity (7). We (9) observed a similar pattern of growth in PT-VLBW infants at 6 and 12 mo corrected age. However, we do not know the age of onset after birth, due to the lack of accurate length measurements before hospital discharge. This has since been addressed, and in recent unpublished observations, it appears that disproportionate growth with a higher $\mathrm{Wt}-\mathrm{L}_{\mathrm{Z}}$ is evident before hospital discharge or as early as 3-4 mo of age in hospitalized PT-VLBW infants. It is unclear if the presence of early disproportionate growth and relative adiposity contribute to the risk for long-term metabolic derangements and elevated SBP; however, in term infants this predicted an elevated SBP at $3 \mathrm{y}$ of age. Thus, feeding practices in the Neonatal ICU, which generally focus on $\mathrm{Wt}$ gain rather than the proportionality of Wt and $\mathrm{L}$ growth, should be examined.

Serum leptin concentrations $>5 \mathrm{ng} / \mathrm{ml}$ are considered elevated in children up to $10 \mathrm{y}$ of age $(28,29)$. Values $\geq 5 \mathrm{ng} /$ $\mathrm{ml}$ were present at $1 \mathrm{y}$ adjusted age in PT-VLBW infants and significantly associated with measures of adiposity, including $\mathrm{Wt}_{\mathrm{z}}, \mathrm{AC}, \mathrm{SS}_{\mathrm{z}}$, and $\mathrm{Wt}-\mathrm{L}_{\mathrm{z}}$ at 1 and $3 \mathrm{y}$. It is unclear why these relationships were not significant at $2 \mathrm{y}$, but similar observations were seen regarding renal function (21). It is possible that the middle cohort represents a transitional period, since $\mathrm{Wt}_{\mathrm{z}}$ did not differ from the reference population whereas the Wt- $\mathrm{L}_{\mathrm{Z}}$ continued to significantly exceed it. Nonetheless, the relationship between elevated serum leptin levels and increased $\mathrm{Wt}$ occurred early. The converse was also observed, i.e., the occurrence of leptin values $\geq 5 \mathrm{ng} / \mathrm{ml}$ decreased with decreasing measures of obesity and/or disproportionate $\mathrm{WtFL}_{\mathrm{z}}$ growth at 1 and $3 \mathrm{y}$ adjusted age. Therefore, serum leptin levels $\geq 5 \mathrm{ng} / \mathrm{ml}$ in infants and toddlers with an elevated leptin/Wt relationship may be a biologic marker for obesity and leptin resistance in PT-VLBW infants as early as 1 y of age. It is unclear, however, if this represents the onset of leptin resistance in the PT-VLBW population and consequently predicts the subsequent development of MBS in later childhood or the adult. If so, it has important implications in the clinical monitoring of PT-VLBW infants. Subsequent studies should prospectively delineate the relationship between various growth parameters and leptin values while in the Neonatal ICU and continue into infancy and late childhood. If correlated, these growth measurements may be useful predictors of future metabolic abnormalities, thereby suggesting the need for changes in care and nutrition during the postnatal period.

The physiologic processes underlying the metabolic derangements that PT-VLBW infants exhibit in later life are not well studied. Studies of the mechanisms underlying childhood and adolescent hypertension in term-born children have posited that cytokines, including leptin, activate the sympathetic nervous system and lead to elevations in blood pressure (30). In adults, this is associated with arterial wall stiffness $(12,17)$. It is notable that there was no relationship between SBP and leptin levels in the present study, particularly considering our earlier observation of an elevated SBP and $\mathrm{SBP}_{Z}$ (9). We (21) reported that, although these PT-VLBW infants demonstrated increasing renal function with advancing age during the first $3 \mathrm{y}$ of life, those with the lowest BW and gestational age had the lowest GFR. Moreover, there was evidence of dysfunctional renal autoregulation, hyperfiltration, and possibly early development of glomerulosclerosis. These findings and those in the current study suggest two possible explanations for the elevated SBP and thus, two populations of PT-VLBW infants, i.e., those at-risk for systolic hypertension with and without MBS. The leptin-mediated mechanism that explains obesity-related hypertension in term-born children, adolescents and adults may occur, but does not account for all cases of hypertension in PT-VLBW children. Infants born $<34 \mathrm{wk}$ of gestation have incomplete nephrogenesis and glomerulogenesis $(31,32)$. Brenner et al. (33) proposed the "nephron number hypothesis", suggesting that when an adverse perinatal environment modifies nephrogenesis, e.g., poor fetal growth or in the present instance, PT birth, there is a nephron deficit due to incomplete nephrogenesis, resulting in decreased renal function and eventually hypertension with glomerulosclerosis. Therefore, the elevated SBP and later hypertension in PT-VLBW children may reflect intrinsic renal deficits, such as incomplete nephrogenesis, rather than leptin mediation (1). It is also unclear to what extent continued glomerular maturation occurs after birth. Notably, recent case reports of glomerulosclerosis in children and adults who were born $<32 \mathrm{wk}$ gestation suggest incomplete development and maturation $(34,35)$. Hence, the elevated SBP may not reflect altered fetal programming, but rather the adverse effects of incomplete renal development associated with very PT birth, which is independent of the early obesity observed in our studies. Detailed longitudinal studies of body composition, leptin levels, renal function, and $\mathrm{SBP}$ in PT-VLBW infants are required to address this. 
Adiponectin levels in healthy subjects decrease in association with increases in adiposity, are inversely related to blood pressure, and increase with weight loss $(11,12,17)$. Serum adiponectin levels in PT-VLBW decreased across cohorts with increasing age, during which time measures of obesity also decreased; e.g., values for $\mathrm{Wt}-\mathrm{L}_{\mathrm{z}}$ in the 3 y cohort did not differ from published norms. This is the opposite of the pattern expected; however, adiponectin levels were inversely related to SBP and mirrored adult correlations. Adiponectin levels decrease in humans and primates with increasing insulin resistance and have a stronger correlation with insulin resistance than adiposity $(36,37)$. Thus, the fall in adiponectin may predict the subsequent development of insulin resistance and MBS. Adiponectin levels also fall in the presence of proinflammatory cytokines (11). Although serum TNF $\alpha$ and IL-6 were unmeasurable and CRP was low and unrelated to any variable examined, other proinflammatory cytokines were not examined. As a result, we cannot determine if an underlying state of chronic inflammation existed in our PT-VLBW infants. If so, the reduced adiponectin levels may be a biomarker for chronic inflammation underlying the elevated SBP in PT-VLBW children without primary renal disease as discussed earlier. This can only be addressed in prospective, longitudinal studies of PT-VLBW infants.

This study has several limitations. The cross-sectional nature does not allow for causal inference or longitudinal comparisons as the measurements were not serial, nor were they obtained in a single cohort. In addition, there may be other biological factors affecting adipokine concentrations apart from adiposity, e.g., sex hormone concentrations and the presence of inflammation (11). Given this, gender findings could have been hormonal in nature rather than a result of adiposity, and proinflammatory illnesses common in children born preterm such as chronic lung disease that were not accounted for could also have impacted adipokine levels. There also could be differences in the PT-VLBW population today that were not present at the time of our study. Finally, the preponderance of Hispanic infants might bias the results in unknown ways, decreasing the generalizability to all PT-VLBW infants. Strengths of the study include the relatively large sample size within each cohort, the multiple anthropometric measurements, and the measurement of adipokines in $>95 \%$ of study infants.

In conclusion, patterns of early growth in infants born PT-VLBW may place them at risk for the development of adiposity and abnormal serum adipokine levels early in infancy. These levels may portend, contribute to, and/or potentiate subsequent development of MBS. The mechanisms responsible for the elevation in leptin levels are unclear. If serum levels are elevated at hospital discharge, as is the Wt-L proportion, feeding practices and immediate postnatal growth may contribute to abnormalities in the synthesis and function of adipokines. Finally, the elevated SBP observed as early as $1 \mathrm{y}$ of age may represent at least two pathogenic mechanisms that cannot be addressed in the present study. Larger serial, longitudinal studies starting soon after birth and extending into late childhood or adolescence are needed to determine how and when these biomarkers change and what, if any, is their relationship to later development of MBS and hypertension in PT-VLBW infants. The current studies should serve as observational studies that are hypothesis-generating.

\section{ACKNOWLEDGMENTS}

This study was supported in part by the George L. MacGregor Professorship held by Rosenfeld. Frankfurt received a Young Investigator Award for the presentation of the leptin data at the 2011 annual meeting of the Pediatric Academic Societies. Thanks to the staff in the Low Birth Weight Clinic for assisting in the conductance of this study.

\section{STATEMENT OF FINANCIAL SUPPORT}

A.F.D. was the recipient of a Marshall Klaus Award from the American Academy of Pediatrics, Section on Perinatal Pediatrics to pursue these studies.

Disclosure: There are no disclosures or conflicts of interest.

\section{REFERENCES}

1. Johansson S, Iliadou A, Bergvall N, Tuvemo T, Norman M, Cnattingius S. Risk of high blood pressure among young men increases with the degree of immaturity at birth. Circulation 2005;112:3430-6.

2. Hofman PL, Regan F, Jackson WE, et al. Premature birth and later insulin resistance. N Engl J Med 2004;351:2179-86.

3. Parkinson JR, Hyde MJ, Gale C, Santhakumaran S, Modi N. Preterm birth and the metabolic syndrome in adult life: a systematic review and metaanalysis. Pediatrics 2013;131:e1240-63.

4. Calkins K, Devaskar SU. Fetal origins of adult disease. Curr Probl Pediatr Adolesc Health Care. 2001; 41:158-176.

5. Vickers MH, Sloboda DM. Leptin as mediator of the effects of developmental programming. Best Pract Res Clin Endocrinol Metab 2012;26:67787.

6. Barker DJ, Osmond C, Forsén TJ, Kajantie E, Eriksson JG. Trajectories of growth among children who have coronary events as adults. N Engl J Med 2005;353:1802-9.

7. Belfort MB, Rifas-Shiman SL, Rich-Edwards J, Kleinman KP, Gillman MW. Size at birth, infant growth, and blood pressure at three years of age. J Pediatr 2007;151:670-4.

8. Duncan AF, Rosenfeld CR, Morgan JS, Ahmad N, Heyne RJ. Interrater reliability and effect of state on blood pressure measurements in infants 1 to 3 years of age. Pediatrics 2008;122:e590-4.

9. Duncan AF, Heyne RJ, Morgan JS, Ahmad N, Rosenfeld CR. Elevated systolic blood pressure in preterm very-low-birth-weight infants $\leq 3$ years of life. Pediatr Nephrol 2011;26:1115-21.

10. Park HK, Ahima RS. Physiology of leptin: energy homeostasis, neuroendocrine function and metabolism. Metabolism 2015;64:24-34.

11. Abella V, Scotece M, Conde J, et al. Adipokines, metabolic syndrome and rheumatic diseases. J Immunol Res 2014;2014:343746.

12. Zachariah JP, Hwang S, Hamburg NM, et al. Circulating adipokines and vascular function: cross-sectional associations in a community-based cohort. Hypertension 2016;67:294-300.

13. Mantzoros CS, Magkos F, Brinkoetter M, et al. Leptin in human physiology and pathophysiology. Am J Physiol Endocrinol Metab 2011;301:E567-84.

14. Mi J, Munkonda MN, Li M, et al. Adiponectin and leptin metabolic biomarkers in chinese children and adolescents. J Obes 2010;2010:892081.

15. Reynolds CM, Gray C, Li M, Segovia SA, Vickers MH. Early life nutrition and energy balance disorders in offspring in later life. Nutrients 2015;7:8090-111.

16. Li S, Shin HJ, Ding EL, van Dam RM. Adiponectin levels and risk of type 2 diabetes: a systematic review and meta-analysis. JAMA 2009;302:179-88.

17. Taylor LE, Sullivan JC. Sex differences in obesity-induced hypertension and vascular dysfunction: a protective role for estrogen in adipose tissue inflammation. Am J Physiol Regul Integr Comp Physiol. 2016; 311: R714-R720.

18. Deng Y, Scherer PE. Adipokines as novel biomarkers and regulators of the metabolic syndrome. Ann N Y Acad Sci 2010;1212:E1-E19.

19. Catalán V, Gómez-Ambrosi J, Rodríguez A, et al. Association of increased visfatin/PBEF/NAMPT circulating concentrations and gene expression 


\section{Articles | Duncan et al.}

levels in peripheral blood cells with lipid metabolism and fatty liver in human morbid obesity. Nutr Metab Cardiovasc Dis 2011;21:245-53.

20. Lambert M, O'Loughlin J, Delvin EE, Levy E, Chiolero A, Paradis G. Association between insulin, leptin, adiponectin and blood pressure in youth. J Hypertens 2009;27:1025-32.

21. Frankfurt JA, Duncan AF, Heyne RJ, Rosenfeld CR. Renal function and systolic blood pressure in very-low-birth-weight infants 1-3 years of age. Pediatr Nephrol 2012;27:2285-91.

22. WHO Multicentre Growth Reference Study Group. WHO child growth standards: length/height-for-age, weight-for-age, weight-for-length, weight-for-height and body mass index-for-age: methods and development. Geneva: World Health Organization, 2006. pp. 312.

23. WHO Anthro for Personal Computers, version 2.0, 2007: Software for assessing growth and development of the world's children. Geneva: WHO. 2006 http://www.who.int/childgrowth/software/en/.

24. WHO Multicentre Growth Reference Study Group. WHO child growth standards: head circumference-for-age, arm circumference-for-age, triceps skinfold-for-age and subscapular skinfold-for-age: methods and development. Geneva: World Health Organization, 2007. pp. 217.

25. Johansson S, Iliadou A, Bergvall N, Tuvemo T, Norman M, Cnattingius S. Risk of high blood pressure among young men increases with the degree of immaturity at birth. Circulation 2005;112:3430-6.

26. Tu W, Eckert GJ, DiMeglio LA, Yu Z, Jung J, Pratt JH. Intensified effect of adiposity on blood pressure in overweight and obese children. Hypertension 2011;58:818-24.

27. Wells JC, Cole TJ. Height, adiposity and hormonal cardiovascular risk markers in childhood: how to partition the associations? Int J Obes (Lond) 2014;38:930-5.
28. Savino F, Rossi L, Benetti S, Petrucci E, Sorrenti M, Silvestro L. Serum reference values for leptin in healthy infants. PLoS One 2014;9:e113024.

29. Erhardt E, Foraita R, Pigeot I, et al.; IDEFICS consortium. Reference values for leptin and adiponectin in children below the age of 10 based on the IDEFICS cohort. Int J Obes (Lond) 2014;38 Suppl 2:S32-8.

30. Flynn JT. Adiposity, the sympathetic nervous system, and childhood primary hypertension. Hypertension 2013;62:689-90.

31. Hinchliffe SA, Sargent PH, Howard CV, Chan YF, van Velzen D. Human intrauterine renal growth expressed in absolute number of glomeruli assessed by the dissector method and Cavalieri principle. Lab Invest. 1994; 64:777-784

32. Gubhaju L, Sutherland MR, Black MJ. Preterm birth and the kidney: implications for long-term renal health. Reprod Sci 2011;18:322-33.

33. Brenner BM, Garcia DL, Anderson S. Glomeruli and blood pressure. Less of one, more the other? Am J Hypertens 1988;1(4 Pt 1):335-47.

34. Hodgin JB, Rasoulpour M, Markowitz GS, D'Agati VD. Very low birth weight is a risk factor for secondary focal segmental glomerulosclerosis. Clin J Am Soc Nephrol 2009;4:71-6.

35. Hibino $\mathrm{S}$, Abe $\mathrm{Y}$, Watanabe $\mathrm{S}$, et al. Proteinuria caused by glomerular hypertension during adolescence associated with extremely premature birth: a report of two cases. Pediatr Nephrol 2015;30:1889-92.

36. Weyer C, Funahashi T, Tanaka S, et al. Hypoadiponectinemia in obesity and type 2 diabetes: close association with insulin resistance and hyperinsulinemia. J Clin Endocrinol Metab 2001;86:1930-5.

37. Lai H, Lin N, Xing Z, Weng H, Zhang H. Association between the level of circulating adiponectin and prediabetes: a meta-analysis. J Diabetes Investig 2015;6:416-29. 